\title{
Treatment of vaginitis and vulvitis
}

\author{
Gayle Fischer, Dermatologist, Westmead, New South Wales
}

\section{SYNOPSIS}

The range of conditions that can cause vulvovaginitis is large and includes infective and non-infective causes. Although Candida albicans is a common infective cause, it is essential to consider other diagnoses. A diagnosis should be made from the clinical presentation, history, bacteriology and in some cases histopathology, before starting treatment. The treatment can then be based on the precise aetiology.

Index words: vulva, vagina, lichen sclerosus, candidiasis.

(Aust Prescr 2001;24:59-61)

\section{Patient presentation}

Women with vulvovaginitis may present with itch, discharge, dyspareunia, burning, soreness, dysuria and swelling. Symptoms may vary with the menstrual cycle. Symptoms are often not a reliable clue to diagnosis, and patients with a variety of different conditions may experience similar symptoms.

\section{Which conditions cause vulvovaginal symptoms?}

Although fungal infections are common they are not the only cause of vulvovaginitis (see Table page 63).

\section{Infections}

C. albicans can cause acute, recurrent and chronic symptoms that always involve the vagina and may also spread to the vulva.

Trichomonas can cause itching and an offensive discharge. Bacterial vaginosis causes a non-irritating discharge.

Group B and sometimes D streptococcus may occasionally cause a vaginitis with a persistent non-offensive discharge, which may cause maceration and irritation of the vulval skin. This infection may co-exist with $C$. albicans.

Herpes simplex does not cause a vulvovaginitis in the true sense. The patient presents with pain and discrete ulcers and blisters on the vulva.

Human papilloma virus causes genital warts. It does not cause itch or discharge.

Tinea is an uncommon cause of vulvitis, but does not cause vaginitis. A scaly rash with a well-defined edge that may extend onto the thighs is characteristic.

\section{Non-infective conditions}

When dealing with women with vulval symptoms remember that disabling pain and burning may occur in the presence of a completely normal vulva and normal bacteriology. These patients do not have vulvovaginitis and may prove to have neuropathic or referred pain. ${ }^{1}$ Rarely is the complaint 'psychogenic'. Despite their lack of apparent abnormality the patient's symptoms should be taken seriously.

\section{Dermatitis}

This is the commonest cause of vulvitis (see picture). It is most often found in atopic individuals, but they may not have a history of dermatitis on other parts of the body. Atopic dermatitis invariably has itch as part of the symptom complex. There is an observable erythematous scaly rash, with fissuring and desquamation involving the labia majora and minora. Atopic dermatitis does not involve the vagina.

Allergic or irritant vulvovaginitis is a dermatitis involving parts of the genital tract that have been exposed to irritating or allergenic substances. This includes imidazole antifungals, neomycin, latex condoms, perfumed oils, overuse of soap and bubble baths, and in rare cases seminal fluid. The dermatitis may be very severe and ulcerative, so that pain and burning are the main symptoms. Urinary and faecal incontinence may also cause a chronic irritant dermatitis.

\section{Psoriasis}

Approximately $2 \%$ of the population has psoriasis. In some individuals it may involve only the genital and perianal skin which can make it hard to diagnose. This itchy and sometimes sore condition does not involve the vagina. There is an erythematous, well-defined rash involving the labia, perianal skin and often the natal cleft.

Vulval dermatitis. The labia majora and minora are erythematous and scaly. There are areas of white discolouration due to lichenification that raise the possibility of vulval intraepithelial neoplasia as a differential diagnosis.

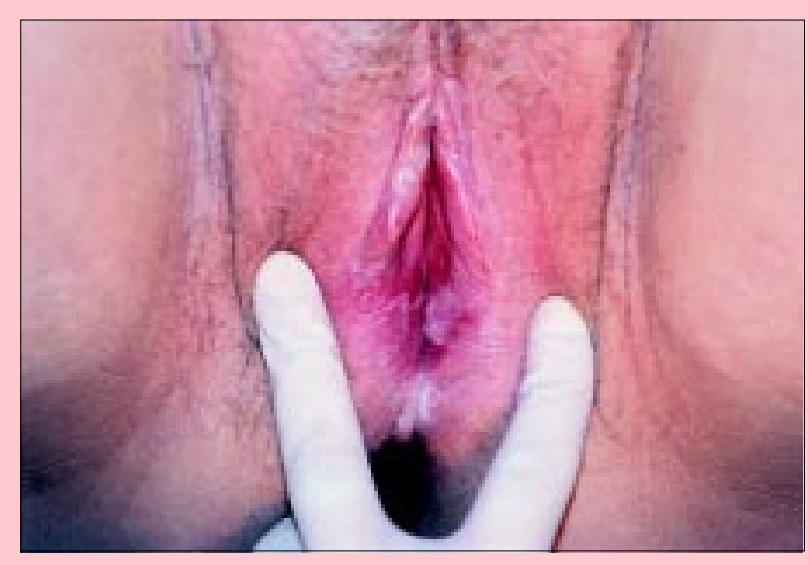

Picture provided by Dr Gayle Fischer 


\section{Lichen sclerosus}

This is a relatively rare, but significant condition. It causes vulvitis only and does not involve the vagina. The very characteristic rash is a white plaque which may involve any part of the vulva or perianal skin. Often there is a telangiectatic or purpuric element. If untreated, shrinkage and scarring of the vulva with loss of the labia and clitoris and stenosis of the introitus may occur. There is a small but real association with carcinoma of the vulva.

\section{Atrophic vaginitis}

Patients who have low oestrogen levels due to menopause or lactation may develop vulvovaginal symptoms of varying severity. The commonest is vaginal dryness which inhibits sexual intercourse. Fragility of the epithelium may result in very small but painful fissures which make intercourse painful. In patients with an underlying atopic diathesis, vulval dermatitis may appear at times of oestrogen deficiency, and in addition to difficulties with sexual intercourse the patient may experience itching or a dermatitic rash. Examination reveals a pale, flat, dry mucosal surface often with tiny fissures around the introitus.

\section{Diagnosis}

As the treatment is based on the precise aetiology of the patient's condition, the first step is to make a correct diagnosis.

\section{History}

A comprehensive history is taken, particularly when symptoms are long-standing. Take the history of the presenting illness, an 'environmental history' (habits, possible allergens and irritants), dermatological history, gynaecological history, general medical and drug history and a psychosexual history.

\section{Examination}

Determine whether a rash is present and whether it is confined to the vulva or also involves the vagina. Occasionally dermatitis and chronic candidiasis may present with no abnormality (often due to recent treatment). However, close inspection may reveal subtle erythema, desquamation or fissuring that are signs of a skin condition. Both psoriasis and lichen sclerosus have characteristic appearances, and diagnosis can often be made on clinical examination.

\section{Bacteriology}

All patients need to have a vaginal swab to rule out $C$. albicans or other pathogens. The pathology lab should be asked to perform microscopy and to report organisms that would ordinarily not be considered to be pathogens. Recent use of antifungal medication will create a false negative, unless the treatment stopped a month before the swab was taken.

Where there is a suspicion of genital herpes, and blisters, erosions or ulcers are present, a viral swab should be taken from the lesion. When an offensive discharge is present a high vaginal swab should be taken.

When the vulva is involved a skin swab should be taken to check for bacterial infection. If tinea is suspected a skin scraping should be taken.

\section{Histopathology}

In some cases the cause of the rash may be elusive or it may be necessary to differentiate conditions that look similar. A biopsy should be taken where possible to confirm lichen sclerosus (because of its serious implications) and where malignancy is suspected.

\section{Treatment}

\section{Dermatitis and psoriasis}

Environmental modification is the first and most essential step, without which treatment is likely to fail. It is also essential to explain the concept of chronicity to these patients, to avoid disappointment if relapse occurs.

Irritating substances (soap, bubble bath, essential oils, antifungal creams, perfumed toilet paper, perfumed sprays) and occlusive clothing (tight jeans, pantyhose, G-strings, nylon underwear and sporting gear, pantyliners, pads) should be abandoned permanently. Cotton underwear, stockings, and tampons rather than pads should be used. A soap substitute is needed. A bland moisturiser that is tolerated by the patient without stinging (vaseline, aqueous cream) can be used daily to reduce dryness and fissuring.

All possible allergens should be eliminated. If there has been a very severe reaction to a suspected allergen, this can be confirmed by applying the substance to the forearm under a band-aid spot for 48 hours, or by formal allergy testing.

When dyspareunia is present, the woman may choose to abstain from sexual activity until she has recovered.

If skin swabs show a clinically relevant infection (most often Staphylococcus aureus), treat with an appropriate antibiotic.

Topical steroids are the treatment of choice for dermatitis and psoriasis. Treatment starts with a potent topical steroid such as methylprednisolone aceponate or betamethasone valerate ointment until the woman is symptom free. Patients must then switch to $1 \%$ hydrocortisone for another month before ceasing treatment. Environmental measures should stay in place indefinitely. Relapses are treated immediately with $1 \%$ hydrocortisone if mild, or a more potent ointment if severe. The patient is instructed to return for review and not to continue to self-medicate if there is no response in a week.

Certain topical steroids (mometasone, aclometasone) are very likely to cause severe stinging on the vulva. They should be avoided.

When using topical steroids on the vulva, remember that longterm use of a potent preparation may eventually cause cutaneous atrophy, striae, and secondary candidiasis. However, usage for a few weeks at a time is safe and is often necessary to induce a remission.

When treating psoriasis, initial management is the same as for dermatitis, but maintenance therapy with $1 \%$ hydrocortisone alone may not be possible. Addition of a tar-containing preparation is the best choice (for example, 2-4\% liquor picis carbonis in aqueous cream). 


\section{Lichen sclerosus}

The treatment of choice for this condition is a superpotent topical steroid such as betamethasone valerate in an optimised vehicle. This is used twice daily for one to two months, then daily until the patient is symptom free, and the white skin changes have reversed. If scarring or fusion is present this will not recover, and it is common to see post-inflammatory hyperpigmentation. Warn the patient that the ointment may sting at first but to persevere as there is no alternative.

Once the patient has improved, maintenance therapy is needed indefinitely. Most patients will require regular daily to weekly use of a moderately potent topical steroid. All patients need to be monitored every six months indefinitely for evidence of squamous cell carcinoma and for the adverse effects of topical steriods.

\section{Atrophic vaginitis}

Treatment of atrophic vaginitis involves the use of topical oestrogen if vulvitis is the only problem. If the patient is experiencing systemic symptoms, hormone replacement therapy is required. Oestrogen creams or pessaries are initially used daily for two weeks then once or twice a week depending on response. There should be an improvement within a month, and if not an alternative diagnosis should be considered. Where there is a concurrent dermatitis, $1 \%$ hydrocortisone ointment should be used daily with a lubricating emollient and a soap substitute. Stronger topical corticosteroids will worsen the atrophy and should be avoided. An oestrogen pessary will be preferable as cream may cause burning where dermatitis is present.

There is no place for the use of oestrogen cream in any condition other than atrophic vulvovaginitis. When used in other situations, oestrogen cream serves only to cause vulval irritation.

\section{Chronic vulvovaginal candidiasis}

This situation is very different from an attack of acute candidiasis, and will not respond to a single course of topical antifungal therapy. These patients have a real problem with eradication of this organism from the vagina. Although in most cases their immunity is quite normal, diabetes and iron deficiency anaemia should be ruled out.

Chronic candidiasis may be a difficult condition to diagnose, as $15 \%$ of women carry Candida in the vagina. Most of these carriers however will be asymptomatic. The combination of chronic vulval symptoms (especially when there is a premenstrual exacerbation or an exacerbation with oral antibiotics) and repeated positive vaginal swabs, is very suggestive of the condition. Examination usually reveals a very inflamed introitus and vagina, with a rash that may spread to the labia. However, sometimes there is very little to see.

When chronic candidiasis is suspected, a trial of therapy with an oral antifungal can be commenced. Although this has not been well researched it is often efficacious and avoids the use of possibly irritating topical antifungals. The only problem is cost, but the results usually justify it. Oral itraconazole, fluconazole or ketoconazole may be used. (The latter is less favoured because of the risk of drug-induced hepatitis.) The medication should be used daily until the symptoms have remitted (anything up to six months) and then weekly for another three months. The addition of $1 \%$ hydrocortisone ointment will help with itch in the early stages.

Many patients find that when they stop therapy relapse occurs. At present we have no answer for this situation other than continued intermittent dosing with oral antifungal medication (weekly to monthly as tolerated).

\section{Summary}

- Always use the history, examination and bacteriological investigation to make a precise diagnosis before treating vulvovaginitis.

- Never assume a patient with vulval symptoms has 'thrush' unless there is a characteristic history supported by positive microbiology.

- Always consider the possibility of a non-infective corticosteroid responsive skin disease, particularly when there is no vaginal involvement.

- Long-term environmental modification is needed when treating vulvovaginal disease.

- Use of potent corticosteroids on the vulva may be a necessary part of treating vulvovaginitis. They may be safely used for limited periods.

E-mail: gaylef@chw.edu.au

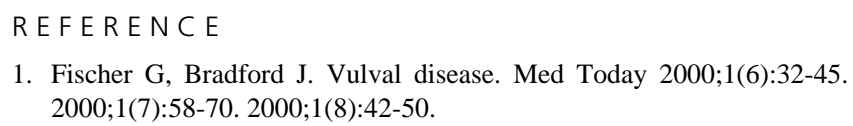

1. Fischer G, Bradford J. Vulval disease. Med Today 2000;1(6):32-45. 2000;1(7):58-70. 2000;1(8):42-50.

\section{FURTHER READING}

Leibowitch M, Staughton R, Neill S, Barton S, Marwood R. An atlas of vulval disease. A combined dermatological, gynaecological and venereological approach. London: Martin Dunitz; 1995.

Sobel JD. Vaginitis. N Engl J Med 1997;337:1896-903.

\section{Self-test questions}

The following statements are either true or false (answers are on page 75)

3. Atopic dermatitis is a common cause of vaginitis.

4. Diabetes should be excluded in women chronically infected with Candida albicans. 